from a high evaluation of the book, conditioned only by the considerations mentioned above.

\title{
Alex Heller
}

Introduction to complex analysis. By Zeev Nehari. Allyn and Bacon, Boston, Mass., 1961. 258 pp. $\$ 7.50$.

It is generally hard to balance a course in complex variables between the theory for the mathematics students and the applications for the engineers. Many universities solve this problem by giving two separate courses. Nehari offers another solution which draws a happy compromise between theory and applications. His book is designed for a one semester course in complex variables (the fashionable word is now complex "analysis") which prepares the interested student for further study in the theory of functions and which is also rich in applications. The book is written in Nehari's informal style which makes enjoyable reading. Embodied in the compromise mentioned above is naturally a sacrifice of generality in some of the basic definitions and theorems, but enough is always given to present a good idea of the general theory of analytic functions.

The chapter titles are: I. Complex variables, II. Analytic functions, III. Complex integration, IV. Applications of Cauchy's theorem, V. Conformal mapping, VI. Physical appliCations, ViI. Additional topics. All of the material that one would expect to find under each of the first six chapter headings is there. There are, however, many little embellishments which make this book especially good. From the very beginning, conjugate coordinates are introduced and used to simplify proofs. There is a nice presentation of the extensions of the elementary functions to the complex plane and their properties. An intuitive "scissors and paste" type of explanation of Riemann surfaces is given. Although the Riemann mapping theorem is stated without proof, the chapter on conformal mapping is especially rich in material for an introductory course. The section on the Schwarz-Christoffel formula is especially noteworthy. The physical applications include boundary value problems, electrostatic fields, and two-dimensional fluid flows. The topics in the last chapter are analytic continuation, the gamma and zeta functions, the symmetry principle, and methods for determining the harmonic conjugate using conjugate coordinates. The book is very richly endowed with stimulating exercises, some of which will certainly challenge the students.

George Springer 